\title{
The role of TREM2 in Alzheimer's disease and other neurodegenerative diseases
}

Susana Carmona, $\mathrm{PhD}^{1,2}$, Kathleen Zahs, $\mathrm{PhD}^{3}$, Elizabeth $\mathrm{Wu}, \mathrm{MS}^{3}$, Kelly Dakin, $\mathrm{PhD}^{3}$, Jose Bras, PhD ${ }^{1,2,4}$, Rita Guerreiro, PhD $^{1,2,4^{*}}$

1. UK Dementia Research Institute at University College London, London, UK

2. Department of Molecular Neuroscience, Institute of Neurology, University College London, London, UK.

3. Alzforum, Cambridge, MA, USA

4. Department of Medical Sciences and Institute of Biomedicine - iBiMED, University of Aveiro, Aveiro, Portugal.

${ }^{*}$ Corresponding author:

Rita Guerreiro, PhD

UK Dementia Research Institute at UCL and UCL Institute of Neurology

Wing 1.2, The Cruciform Building, Gower Street, London WC1E 6BT

P: +44 (0)20 76798142

Email: r.guerreiro@ucl.ac.uk

\section{Abstract}

Alzheimer's disease (AD) is a genetically complex disorder, and rare variants were recently identified in the TREM2 gene that as much as triple an individual's risk of developing AD. TREM2 is a transmembrane receptor expressed in cells of the myeloid lineage, and the association with $A D$ confirmed the involvement of immune and inflammatory pathways in the etiology of the disease, as opposed to a reaction. TREM2 variants associated with AD induce partial loss of function of TREM2 protein and alter the behavior of microglial cells, including their response to amyloid plaques. TREM2 variants have also been found to cause polycystic lipomembranous osteodysplasia with sclerosing leukoencephalopathy and FTD. Although the low frequency of TREM2 variants makes it is difficult to establish robust genotype-phenotype correlations, such studies are essential to enable a more comprehensive understanding of the role of TREM2 in different neurological diseases, with the ultimate goal of developing novel therapeutic approaches. 


\section{Introduction}

Alzheimer's disease (AD) has a complex genetic architecture with rare, highly penetrant, mutations in the amyloid precursor protein (APP), presenilin-1 (PSEN1) and presenilin-2 (PSEN2) genes, $\sim 30$ common low risk genetic loci ${ }^{1}$ and the $\varepsilon 4$ allele of $A P O E$ which has an intermediate frequency in the population and imparts intermediate risk for disease. Identifying additional genetic variants with frequencies and effects similar and lower to the ones conferred by $A P O E$ has been exceedingly difficult given the need for extremely large cohorts of cases and controls. However, these variants have the potential to add significantly to our understanding of the biology of the disease process.

Whole genome analyses led to the identification of a rare variant in TREM2 - a gene that encodes a receptor expressed in myeloid cells that mediates inflammatory responses (panel) imparting a risk for $A D$ similar to that conferred by one copy of the APOE $\varepsilon 4$ allele (increasing risk for $A D$ by approximately 3 -fold $)^{2,3}$. The importance of TREM2 in brain function is also highlighted by the fact that rare, biallelic mutations in the gene cause polycystic lipomembranous osteodysplasia with sclerosing leukoencephalopathy (PLOSL, also known as Nasu-Hakola disease) ${ }^{4}$ and, in some cases, frontotemporal dementia (FTD) ${ }^{5}$. Understanding the biological functions of TREM2 in the brain is essential for the identification of potential therapeutic targets and, possibly, new candidate genes ${ }^{6}$.

In this Review, we discuss the reported genetic associations of TREM2 with neurodegenerative diseases, as well as the effects of these variants on pathology, clinical presentation, and underlying biology. We cover AD, PLOSL, and FTD, with an emphasis on AD, as it is the most common neurological disease associated with TREM2, and many advances in understanding TREM2's role in the brain have been made in AD. Specifically, we assess the evidence for the association of identified TREM2 variants with AD risk, summarise genotype-phenotype correlations, evaluate the potential use of soluble TREM2 (sTREM2) as a CSF biomarker, and summarise the biological effects of loss of TREM2 function. We underscore the importance of integrating multi-disciplinary findings related to TREM2 and introduce an on-line resource at Alzforum that aggregates existing and emerging results pertaining to neurological disease.

\section{TREM2 in Alzheimer's disease}

\section{TREM2 variants and AD risk}

So far, a total of 63 coding genetic variants have been identified in TREM2 with varying frequencies in the general population. One of these, a rare variant ( $p . R 47 \mathrm{H}, \mathrm{rs} 75932628)$ has been found to increase the risk of developing AD by 2 to 3-fold in several European and NorthAmerican populations ${ }^{2,3,7-10}$. A significant association with AD between a proxy of rs75932628 and $A D$ was also found in black patients ${ }^{11}$. However, in Chinese cohorts, p.R47H was not detected in patients with late-onset $A D$ or healthy controls ${ }^{12-15}$ and no association between TREM2 variants and AD risk was found in Japanese ${ }^{16}$, Korean ${ }^{17}$ or Iranian ${ }^{18}$ cohorts, which suggests that effect of TREM2 is population-specific. 
Studies in family based datasets found a weak but significant association between p.R47H and AD risk ${ }^{19,20}$. In a study ${ }^{21}$ of 130 families with late-onset $A D, T R E M 2$ p.R47H was found in 4 families (3\%, a value 10 times higher than that in the general population) and in a large family with late-onset $A D, 12$ patients $(75 \%)$ with $A D$ were found to carry the p.R47H allele, with vertical transmission of the disease observed only through p.R47H carriers.

Other TREM2 variants have also been tested for association with AD risk (appendix, for an up to date interactive representation of mutations in TREM2 see

https://www.alzforum.org/mutations) with only p.R62H showing genome-wide association levels of significance ${ }^{7}$ and shown to be independent of the p.R47H association ${ }^{8}$. Two studies of Chinese cohorts revealed two novel variants p.A130V ${ }^{14}$ and $p . A 192 \mathrm{~T}^{22}$ exclusively in patients with late-onset $A D$. The variants p.H157Y and p.S183C were more frequent in Chinese patients with $A D$, with p.H157Y being significantly associated with increased risk for late-onset $A D^{22}$, contrary to what had been found in Caucasian cohorts. Subsequent functional studies have added evidence to the involvement of this variant in AD by identifying amino acid 157 as the site of protein shedding with $\mathrm{p} . \mathrm{H} 157 \mathrm{Y}$ accelerating the cleavage process and resulting in less fulllength protein on microglia ${ }^{23,24}$. In the African American population only the variants p.W191X and p.L211P showed suggestive associations with risk for late-onset $A D{ }^{25}$. The identification of different variants associated with risk for $A D$ in the Chinese and African Americans shows the importance of studying the association of rare variants with disease in different populations, as mutations occur at different rates across different populations, leading to differences in the spectrum of segregating polymorphisms.

In addition to TREM2 variants, other changes located in the TREM gene cluster have been reported to be associated with $A D$ risk or to regulate TREM genes expression. An intergenic SNP located 5.5Kb downstream from TREML2 and $24 \mathrm{~Kb}$ upstream from TREM2 (rs9381040) was shown to act as a protective allele for AD ${ }^{26}$. Similarly, a TREML2 missense variant (p.S144G, rs3747742) was also found to reduce the risk for $A D^{27}$. The association of these two variants appears to be independent of $p . R 47 \mathrm{H}^{27}$. An association with reduced risk of $A D$ was reported for rs9357347, located within a DNase hypersensitive site between TREM2 and TREML2 ${ }^{28}$. This variant is in linkage disequilibrium with rs9381040 and the authors considered rs9357347 as a regulatory and functional variant, since it was also associated with increased TREML 1 and TREM2 mRNA levels ${ }^{28}$.

Having variants that are robustly associated with $A D$ and have appreciable effect sizes opens the possibility to genetic risk prediction, particularly when vertical transmission has been observed. However, the effect sizes of these variants are too low to allow risk prediction. Although the p. $\mathrm{R} 47 \mathrm{H}$ variant has an odds ratio that is comparable to one copy of the $\varepsilon 4$ allele in $A P O E$ - on its own, is not helpful in clinical risk prediction. Additionally, given the rarity of these variants it is unlikely they will have an impact on risk prediction at the population level, but it may be useful to include TREM2 variants in polygenic risk predictions for $A D$ in a small subset of cases. None of these variants are disease-causing and, with the exception of p. R47H and p. $\mathrm{R} 62 \mathrm{H}$, all other associations still need to be independently replicated by large cohort studies.

\section{Genotype-phenotype correlation}


Several studies have tried to correlate the presence of TREM2 variants with AD-related phenotypes, but so far too few carriers have been studied to reach definite conclusions. In general, patients with AD carrying TREM2 variants show similar clinical, neuroimaging and neuropathology features to those seen in sporadic cases $2,21,29$. In a clinic-based dataset of 563 patients with $A D, 12$ were carriers of p.R47H and have been reported to have lower age at onset when compared to 551 non-carrier patients with $A D$ (mean: 55.2 years vs. 61.7 years), respectively ${ }^{29}$. In fact, most of these 12 carriers of p.R47H had an age at onset below 65 years, with 4 below 50 years and no other known genetic risk variants. These results are consistent with those reported for the Icelandic and Dutch populations in whom the presence of the risk allele led to an age at onset of AD that was lower by 3.18 and 3.65 years than in non-carriers of the variant, respectively ${ }^{21}$. However, in five families with lateonset AD, when comparing the age of onset of p.R47H carriers $(n=16)$ with non-carriers $(n=18)$, the mean did not differ between groups. In these same families, disease duration was significantly shorter in carriers than in non-carriers ${ }^{21}$.

In a cohort of asymptomatic middle-aged adults, p.R47H carriers had a higher probability of having a parental history of $A D$, with a younger maternal age of $A D$ onset; while no differences were observed in cognitive function or rate of decline ${ }^{37}$. However, in the Icelandic population, non-demented carriers with ages between 80 and 100 years exhibited poorer cognitive function than non-carriers of $\mathrm{p} . \mathrm{R} 47 \mathrm{H}^{3}$.

The presence of TREM2 p.R47H showed no association with psychosis, amyloid deposition in the brain, or family history ${ }^{9,30}$. Higher density of $A \beta$ plaques and neurofibrillary tangles ${ }^{31}$ and more frequent $\alpha$-synucleinopathy ${ }^{21}$ have also been observed in patients with TREM2 p.R47H. In a Spanish cohort, AD carriers of p.R47H showed apraxia, psychiatric symptoms such as personality changes, anxiety, paranoia or fears and parkinsonian signs more frequently than $A D$ non-carriers, particularly during the first 2 years after disease onset ${ }^{32}$. Higher frontobasal gray matter cortical loss was also reported in p.R47H carriers in this cohort, which could be speculatively related to the FTD phenotype associated with some TREM2 variants ${ }^{32}$. Patients with TREM2 p.R47H were also found to have significantly higher levels of total tau and phosphorylated tau ( $\mathrm{p}$-tau) in CSF, whereas $A \beta 42$ levels were comparable to expected concentrations for the stage of disease ${ }^{33,34}$. When compared to non-carrier controls, $A D$ patients carriers of p.R47H were also found to have higher CSF sTREM2 levels ${ }^{35}$. In the same study p.R62H showed no difference to controls but other TREM2 variants were found to be associated with lower levels of CSF sTREM2 with the lowest levels identified in carriers of heterozygous PLOSL-causing variants ${ }^{35}$. By studying TREM2 rs9394721 (a proxy for p.R47H) in the Alzheimer's Disease Neuroimaging Initiative cohort, it was found that variant carriers annually lost $1.4 \%$ to $3.3 \%$ more of their brain tissue than non-carriers, in a pattern that mirrored the profile of $A D$ in the brain. ${ }^{42}$ The risk allele was also found to be significantly associated with smaller hippocampal volumes, elevated levels of the CSF p-tau, and poorer cognitive performance compared to non-carriers ${ }^{36}$.

Given that leukoaraiosis is commonly seen in patients with PLOSL it is interesting to note that no significant changes in white matter have been described for AD patients with TREM2 variants ${ }^{32}$. No differences between carriers and non-carriers of p.R47H have been seen when using the Scheltens scale (semi-quantitative visual rating scale used to measure the presence of hyperintense white matter lesions in MRI) or when looking specifically for clusters of white 
matter hyperintensities ${ }^{32}$. Similarly, neuropathology assessment of brain tissue from AD carriers of TREM2 variants showed white matter abnormalities within what would be expected for typical $A D^{2}$.

Variants located in the TREM locus have also been associated with AD-related phenotypes. For example, TREML2 variants p.S144G and rs6916710, and the intergenic variant rs6922617 were found to be associated with CSF p-tau levels ${ }^{27,34}$. The TREM1 intronic variant rs6910730 was found to increase the burden of neuritic and diffuse plaques, as well as of $A \beta$ density, while rs7759295 was associated with increased tau tangle density and increased burden of neurofibrillary tangles ${ }^{38}$. Both variants were associated with an increased rate of cognitive decline ${ }^{38}$. It is plausible that many, or even all, of the variants reported with correlations with AD-associated phenotypes, may be in linkage disequilibrium with the functional variant and consequently may reflect indirect associations.

Larger cohorts and replication studies are required to enable robust genotype-phenotype correlations. The available data suggest that AD patients carriers of TREM2 variants are similar to typical patients with AD with an initial amnesic syndrome, but perhaps presenting earlier age at onset and faster loss of brain tissue (particularly in frontobasal cortical regions) which is possibly associated with a more rapid progression into multidomain dementia. Although these findings do not have, as of yet, a direct impact in clinical practice, identifying carriers of TREM2 variants may be important in patient stratification for clinical trials and future individualised therapeutic approaches.

\section{sTREM2 as a CSF biomarker}

sTREM2 is released to the extracellular space after proteolytic cleavage and can be detected in human plasma and CSF in both healthy and AD subjects ${ }^{39}$. Even though the physiological and disease-associated functions of sTREM2 are unknown, this form of the protein has been suggested to have protective effects on microglial cell viability ${ }^{40}$. Additionally, AD-risk associated variants were shown to reduce the overall capacity of sTREM2 to enhance cell viability and to trigger inflammatory responses in microglia ${ }^{40}$. Although the physiological relevance of these results is not clear, it is interesting to consider that sTREM2 may act independently, and possibly with opposing roles, to the full-length protein in the regulation of inflammatory responses.

Although the initial studies ${ }^{39,41}$ examining the differences in CSF sTREM2 levels between patients with $A D$ and healthy individuals did not show any differences ${ }^{39}$ or showed reduced levels in patients with $A D$ compared to healthy controls ${ }^{41}$, subsequent studies have consistently found higher levels of CSF sTREM2 in patients with AD compared to healthy controls ${ }^{35,42,43}$. These apparently contradictory findings may stem from differences in the assays used or from the focus on different stages of disease progression. CSF sTREM2 levels change during disease progression with a peak at the clinical $\mathrm{MCl}$ stage of $\mathrm{AD}{ }^{43}$. In a cross-sectional study ${ }^{44}$ of individuals enrolled in the Dominantly Inherited Alzheimer's Network, carriers of PSEN1, PSEN2, or APP AD-causative mutations were found to have elevated levels of CSF sTREM2 compared with non-carriers; this difference became apparent five years before the expected onset of symptoms in the mutation carriers and remained significant until five years after expected onset. In this cohort, elevations in CSF sTREM2 followed brain amyloidosis and 
elevations of CSF tau, and were concurrent with the presence of cerebral hypometabolism and hippocampal atrophy ${ }^{44}$. Other studies revealed that levels of CSF sTREM2 positively correlate with levels of CSF p-tau and total-tau, ${ }^{35,39,42,43}$ but not with CSF A $342{ }^{35,42}$. These findings suggest a dynamic response of STREM2 during the disease process, that may reflect an association between microglial activity and the first signs of degeneration ${ }^{43,44,45}$.

Despite the significant association between CSF sTREM2 levels and AD at the group level, CSF STREM2 only achieves a discriminative power of around $60 \%$, which is lower than the $80-90 \%$ required to be useful in clinical diagnostic procedures ${ }^{46}$. Nonetheless, if CSF sTREM2 levels are reproducibly associated with the onset of cognitive decline,CSF sTREM2 could provide an independent measure of disease stage, marking the transition from preclinical AD to dementia, and could be useful for clinical trials.

\section{TREM2 mechanisms in AD}

The identification of TREM2 variants as risk modifiers for $A D$ stimulated research into the pathobiological mechanisms mediated by this microglial receptor. Neuropathology in patients with $A D$ and animal models, coupled with in vitro studies of the biochemical and cell biological effects of TREM2 variants, indicate that AD-associated risk variants cause partial loss of function of the protein.

As the resident immune cells of the brain, microglia continuously survey the brain to respond to foreign invaders, while providing trophic support for neurons and phagocytosing cellular debris 47. Microglia surround amyloid plaques in the brains of patients with $A D$ and of animal models of amyloidosis, and it has been suggested that these microglia form a physical barrier that encapsulates neurotoxic amyloid- $\beta^{48,49}$. Fewer plaque-associated microglia were seen in patients heterozygous for the p.R47H and p. $\mathrm{R} 62 \mathrm{H}$ variants compared with the brains of patients without TREM2 risk variants ${ }^{50}$, and more severe plaque-associated neuritic dystrophy accompanied reduced microglial coverage in the brains of $p . R 47 \mathrm{H}$ patients with $A D{ }^{48}$. The effect of the $p . R 47 \mathrm{H}$ variant on microglial clustering around plaques was recapitulated in a mouse model of amyloidosis in which the mouse Trem2 gene was replaced with either the wildtype or p.R47H variant of human TREM $2^{51}$.

The role of TREM2 in the context of amyloid pathology has been further studied by manipulating TREM2 gene dosage in mouse models of amyloidosis. Fewer microglia surrounded plaques in animals lacking or haploinsufficient for TREM2 ${ }^{49,52}$, similar to what is seen in the brains of patients with $A D$ carrying the p.R47H or p.R62H alleles, suggesting that the AD-associated variants are partial loss of function mutations. Plaque-associated neuritic dystrophy was also more severe ${ }^{48,49}$ and neuron loss was exaggerated in mice deficient for TREM2, in a gene-dose dependent manner ${ }^{53}$. The effects of TREM2 dosage on amyloid burden are more complex. Genetic ablation of TREM2 resulted in less severe plaque pathology at early stages of plaque deposition but in more severe plaque pathology at later stages ${ }^{49,52-54}$. Elevating levels of TREM2 through the introduction of a human TREM2 transgene reduced pathology in amyloidbearing mice ${ }^{55}$. Specifically, amyloid burden was reduced and plaques were remodeled from a fibrillary type to a more compact and possibly less toxic form. Furthermore, microglial phagocytic activity was increased, the number of plaque-associated microglia was reduced, and cognitive function was rescued ${ }^{55}$. 
The biochemical and cell biological effects of $A D$-associated variants in TREM2 have been studied extensively in vitro. AD-associated TREM2 variants do not significantly affect the folding, trafficking, or expression of TREM2 $41,53,56,57$, but do impair ligand binding and uptake $7,41,53,57-62$. Although the endogenous ligands of TREM2 are not known, molecules that act as TREM2 ligands under experimental conditions - and that display reduced binding to ADassociated TREM2 variants-include anionic or zwitterionic lipids that may be exposed during neuronal and glial degeneration and associate with fibrillar amyloid- $\beta^{53}$, ApoE ${ }^{60,61}$, and oligomeric amyloid- $\beta^{58}$.

Two theories have emerged to explain how the loss of TREM2 function might impair the microglial response to amyloid pathology and exacerbate disease: 1) TREM2 signaling is necessary to reprogram cells from a homeostatic to a neuroprotective disease-associated phenotype ${ }^{50,63,64}$, and 2) tonic TREM2 signaling is necessary to support microglial metabolism, and loss of TREM2 function severely impairs microglial fitness and capacity to respond to stressors ${ }^{65}$.

In support of the first theory, plaque-associated microglia adopt a gene expression profile distinct from that of microglia in healthy brain (homeostatic microglia), characterized by increased expression of genes associated with lipid metabolism and phagocytosis ${ }^{50,63}$. TREM2 is necessary for microglia to undergo this phenotypic switch ${ }^{50,63}$. It has been suggested that the plaque-associated microglial phenotype may be neuroprotective, promoting phagocytosis of amyloid plaques and/or degenerating neurites ${ }^{63}$, and that microglia locked into a homeostatic state could be detrimental ${ }^{64}$. In support of the second theory, TREM2-deficient microglia have abundant autophagosomes, believed to indicate metabolic stress, and decreased expression of genes involved in biosynthesis and energy metabolism - a state very different from homeostatic microglia ${ }^{65}$. Notably, augmenting ATP levels with dietary cyclocreatine ameliorated the effects of TREM2 deficiency on the microglial response to plaques in amyloid-bearing mice, providing support for the hypothesis that metabolic deficits underlie the effects of TREM2 loss of function on at least some aspects of AD-related pathology 65 .

The mechanisms through which TREM2 influences AD may be multiple and complex. It is possible that TREM2-mediated microglial activity might be both protective and damaging, depending on the stage of disease progression and the specific microglial activity (e.g., phagocytosis of potentially damaging material versus generation of potentially damaging cytokines). To date, only very few studies have examined the effects of TREM2 deficiency in the context of tauopathy and no consistent picture has yet emerged ${ }^{66-68}$. The availability of new animal models expressing disease-associated TREM2 variants and of induced pluripotent stem cells derived from patients with AD carrying TREM2 mutations should accelerate research aimed at better defining the role of TREM2 in the development and progression of AD.

\section{TREM2 mutations cause PLOSL}

PLOSL, also known as Nasu-Hakola disease, is a very rare, autosomal recessive disease characterized by spontaneous bone fractures and early-onset dementia ${ }^{4}$. In Finland, it has an estimated prevalence of 1 to 2 per million people ${ }^{69}$. It has been diagnosed in over 100 cases in the Japanese population ${ }^{69}$ and appears to be less common in other all other worldwide 
populations ${ }^{70}$ with the first report from India just recently published ${ }^{71}$. It is a genetically heterogeneous syndrome caused by biallelic (homozygous or compound heterozygous) mutations in two genes-TREM2 or TYROBP ${ }^{4}$. The natural course of the disease can be divided into four stages: latent, osseous, early neurological, and late neurological ${ }^{72}$ (appendix). Several other mutations in the gene have been reported to cause PLOSL (appendix). In the first report linking TREM2 mutations with PLOSL, of the 39 patients studied, TYROBP mutations were identified in 31 (79\%) patients and TREM2 mutations in the remaining $8(21 \%)$ patients ${ }^{4}$. Note that 25 of these patients carried the same $5.3 \mathrm{~kb}$ deletion encompassing exons 1-4 of $T Y R O B P^{4}$ suggesting that this is a founder mutation in the Finnish population and should be tested for specifically in Finnish patients. The genetic study of a Finnish family harbouring this TYROBP deletion revealed the co-occurrence of other variants in neurologically-associated genes, including a C9ORF72 expansion which was thought to be an incidental finding with no clinical expression (most likely due to the higher frequency of both genetic changes in this population), and a novel mutation in EPM2A, a gene implicated in progressive myoclonic epilepsy type 2 (Lafora disease), that was suggested to be responsible for the severe epilepsy seen in the corresponding case of this Finnish family ${ }^{73}$. AD pathology has also been studied in PLOSL patients. Amyloid-PET revealed extensive A $\beta$ deposition in the grey matter of the inferior frontal and occipital lobes of an Italian patient carrying a TREM2 homozygous p.Q33X mutation 74 . Neuropsychological and functional nuclear imaging ( ${ }^{99 m}$ Tc-ECD SPECT) tests in heterozygotes in a family carrying this same mutation showed deficits of visuospatial memory associated with hypoperfusion in the basal ganglia implying an overlap of pathogenic mechanisms between AD and PLOSL ${ }^{75}$. However, immunohistochemistry analyses of five brains of patients with PLOSL revealed no amyloid plaques or amyloid angiopathy, and only a small number of tau-tangle bearing neurons, mostly in the hippocampus, suggesting that TREM2 loss of function does not accelerate AD pathology ${ }^{76}$. Additionally, analyses of TYROBP genetic variability have also not shown associations with cognitive impairment in a Finnish cohort ${ }^{77}$ or as the cause of dementia in Turkish patients ${ }^{78}$.

The implication of TYROBP and TREM2 mutations in PLOSL led to the identification of the signaling pathway ${ }^{4}$ responsible for this disease. The finding that these genes are both expressed in microglia and osteoclasts partially explained the peculiar tissue distribution of symptoms. However, it is still unclear why some patients (sometimes harbouring the same mutation) develop bone cysts and pathological fractures associated with dementia, while others present with FTD (for an overview of phenotype by mutations, see Supplementary Figure 3). It is tempting to speculate that genetic modifiers play a role in these disparate phenotypes, however achieving large enough sample sizes to identify them for variants that have such a low frequency in the population will be challenging. Despite this, it would be important to understand which factors influence these differences in presentation as these may be protective and potential targets for preventive or symptom management therapies.

\section{TREM2 in frontotemporal dementia}

Biallelic TREM2 mutations have also been described in 10 families diagnosed with FTD without the PLOSL bone phenotypes ${ }^{79-86}$ (appendix). Some of these variants were previously known to cause PLOSL, while others are newly identified causes of disease. There seems to be no 
correlation between the type of mutation and the associated phenotype since nonsense, splice site, and missense mutations have now been shown to cause both diseases ${ }^{81}$. For a discussion of the mechanisms involved in PLOSL and FTD caused by TREM2 mutations, see appendix. Dementia in the form of an early-onset personality change, resembling behavioural variant FTD, seems to be the most common feature of all the reported FTD cases with biallelic TREM2 mutations. A family history of consanguinity together with atypical features, such as seizures and corpus callosum atrophy, in a patient presenting with a behavioural FTD phenotype, should prompt the diagnostician to look for biallelic TREM2 mutations. This genetic cause should also be included in the differential diagnosis of young-onset dementia patients with seizures ${ }^{81}$. Novel heterozygous TREM2 variants in patients with suggestive clinical presentations should be critically assessed and not assumed to be disease causative. Only in the presence of informative co-segregation of the variant with disease in a family, or unbiased functional effects in cell biology assays resulting in meaningful phenotypes should such variants be considered as disease causing. Association of TREM2 heterozygous variants with risk for FTD is currently controversial with several contradictory results reported. The p.T96K variant was associated with FTD subtypes ${ }^{87}$ and a substantial burden of TREM2 heterozygous variants was reported in patients with FTD when compared to healthy controls ${ }^{10,88}$. The $p . R 47 \mathrm{H}$ variant was found to be associated with FTD risk in a North American cohort ${ }^{89}$ but not in European cohorts ${ }^{9,29,90}$. Furthermore, a large meta-analysis of patients carrying p.R47H did not show a significant association with risk for FTD ${ }^{33}$. These findings point to a possible association of TREM2 with the risk of FTD at the gene level, but without any reliable associations at the variant level. These inconclusive results are likely due to the rarity of TREM2 variants and small cohort studies with not-well characterised cases.

\section{TREM2 genetic variability in other neurodegenerative diseases}

Given the role of TREM2 in microglial function and its involvement in three neurodegenerative disorders (AD, FTD, and PLOSL), it has been suggested that TREM2 could be part of a functional network involved in various neurodegenerative diseases ${ }^{91}$. Consequently, several studies have assessed the association of TREM2 variants with risk of developing non-AD neurodegenerative disease (appendix).

In 2013, after the initial findings of the association between TREM2 and AD, an association of p. R47H with the risk of Parkinson's disease (PD) was reported ${ }^{91}$. However, in this study, the frequency of the variant in healthy controls was below what would be expected given known population frequencies. Since the $\mathrm{p} . \mathrm{R} 4 \mathrm{HH}$ variant frequency varies across populations, it is imperative that, for this and other rare variants, association tests be performed only in sufficiently large and well-matched case-control groups ${ }^{92}$. In another study, a significant association between p.R47H and PD risk in a discovery cohort of North American patients diagnosed with PD and healthy controls was reported, but failed replication in that same study ${ }^{89}$ The association of p.R47H with PD has subsequently failed independent replication in other 
association studies ${ }^{33,93}$. This association was also tested in the Chinese population and no significant effect on risk was found, again, with no variant being identified in this population ${ }^{94-97}$. Other studies have evaluated the impact of TREM2 variants in risk for amyotrophic lateral sclerosis ${ }^{89,98,99}$, Lewy body dementia ${ }^{100}$, posterior cortical atrophy ${ }^{101}$, Creutzfeldt-Jakob disease ${ }^{9,29,90}$, progressive supranuclear palsy ${ }^{89}$, ischemic stroke ${ }^{89}$, multiple system atrophy ${ }^{95}$, and essential tremor ${ }^{102}$, with conflicting results (appendix). This is, at least in part, because the TREM2 variants are rare and only small cohorts of patients have been analysed. Thus, the association of TREM2 with the risk for the development of neurodegenerative diseases beyond AD has not been robustly replicated so far. Future studies comprising larger cohorts of cases and controls will be required to clarify these potential associations.

\section{Conclusions and future directions}

Although the associations of TREM2 p.R47H and p.R62H variants with risk for AD are well established, more studies are required to validate the possible associations of other variants within the TREM2 gene, as well as those located within the broader TREM locus. The same is true for variants in other neurodegenerative diseases: thus far, studies have yielded contradictory results, with the burden of proof not being met. Large-scale studies, preferably including populations other than European-Americans, are necessary to more fully understand the impact of TREM2 variability on the genetic architecture of $A D$ and other neurodegenerative disorders.

Additionally, further large-scale studies are needed to better understand the impact of TREM2 variants on clinical presentation, progression, and neuropathology. A limited number of studies thus far suggest that $A D$ patients carrying p.R47H present with fairly typical AD symptoms, although earlier age of onset and shorter disease duration have been reported ${ }^{21,29}$. In addition, reduced microglial clustering around plaques was seen at autopsy in p.R47H carriers ${ }^{48,50}$. However, given the low frequency of TREM2 variants, it is difficult to assemble sufficiently large cohorts to establish robust genotype-phenotype correlations.

Although sTREM2 has been found to be elevated in the CSF of patients with AD, compared with controls, it lacks discriminative power for diagnostic procedures ${ }^{46}$. However, CSF sTREM2 appears to exhibit a dynamic response during disease progression ${ }^{43,44}$, possibly reflecting microglial activity. If validated, STREM2 could be a potential biomarker for disease progression, particularly the transition from preclinical $A D$ to dementia. It is interesting to imagine the use of amyloid- $\beta$ and tau biomarkers for the differential diagnosis of AD, and a panel of other biomarkers, including STREM2, to characterise and stage the disease.

Additional research is also needed into how TREM2 variants affect risk of AD and cause PLOSL and FTD. Evidence thus far suggests that TREM2 variants that result in (partial) loss of protein function may impair microglial response to stressors, such as amyloid accumulation in $A D{ }^{65}$. Evidence points towards TREM2 as a potential therapeutic target. In vivo TREM2 overexpression ameliorates neuropathological signatures of $A D$ and the memory deficits associated with the disease ${ }^{55}$. Thus, activation of the TREM2 signaling pathway could offer a new therapeutic approach, for example, through the use of small molecules that mimic TREM2 ligands. It is also important to determine when, during the course of the disease, an intervention 
increasing TREM2 expression or activation might be most beneficial. The study of familial forms of $A D$ can potentially be helpful for this goal, due to the predictability of the age of onset of symptoms. It will also be important to systematically characterize the effects of manipulating TREM2 levels, both centrally and peripherally.

In order to fully understand the complex role of TREM2 in the risk of neurodegenerative diseases it is essential to systematically integrate the available data. To aggregate the existing TREM2 variant data and provide a platform into which new information can be synthesized, we have created a TREM2 open access resource on the Alzforum website (www.alzforum.org/mutations) - in which information on genetic associations with different phenotypes, pathogenicity, neuropathology, biological effects, and research models, are available for identified TREM2 variants. This resource currently contains information on 63 TREM2 variants and will be updated as new findings are published.

A significant increase in the number of studies related to TREM2 in neurodegenerative diseases and the critical integration of these results will allow us to understand its role in neurodegenerative disorders, which will facilitate the development of new therapeutic interventions.

\section{Contributors}

All authors contributed equally to the preparation and writing of the manuscript. All authors approved the final version.

\section{Declaration of interests}

All the authors declare no competing interests.

\section{Acknowledgments}

JB and RG's work is funded by research fellowships from the Alzheimer's Society.

\section{Search strategy and selection criteria}

References for this Review were identified by searches of PubMed and BioRxiv databases for peer-reviewed, English articles published from Jan 1, 2013, to May 31, 2018. The search terms 'TREM2 AND dementia', 'TREM2 AND Alzheimer' and 'TREM2 AND neurodegeneration' were used. The final reference list was generated on the basis of relevance to the topics covered in this Review.

\section{References}

1 Jansen I, Savage J, Watanabe K, et al. Genetic meta-analysis identifies 9 novel loci and functional pathways for Alzheimers disease risk. bioRxiv. 2018; : 258533. 
2 Guerreiro R, Wojtas A, Bras J, et al. TREM2 variants in Alzheimer's disease. N Engl J Med 2013; 368: 117-27.

3 Jonsson $\mathrm{T}$, Stefansson $\mathrm{H}$, Steinberg S, et al. Variant of TREM2 associated with the risk of Alzheimer's disease. N Engl J Med 2013; 368: 107-16.

4 Paloneva J, Manninen T, Christman G, et al. Mutations in two genes encoding different subunits of a receptor signaling complex result in an identical disease phenotype. $A m \mathrm{~J}$ Hum Genet 2002; 71: 656-62.

5 Guerreiro RJ, Lohmann E, Brás JM, et al. Using exome sequencing to reveal mutations in TREM2 presenting as a frontotemporal dementia-like syndrome without bone involvement. JAMA Neurol 2013; 70: 78-84.

6 Forabosco P, Ramasamy A, Trabzuni D, et al. Insights into TREM2 biology by network analysis of human brain gene expression data. Neurobiol Aging 2013; 34: 2699-714.

7 Song W, Hooli B, Mullin K, et al. Alzheimer's disease-associated TREM2 variants exhibit either decreased or increased ligand-dependent activation. Alzheimers Dement 2017; 13: $381-7$.

8 Sims R, van der Lee SJ, Naj AC, et al. Rare coding variants in PLCG2, ABI3, and TREM2 implicate microglial-mediated innate immunity in Alzheimer's disease. Nat Genet 2017; 49: 1373-84.

9 Ruiz A, Dols-Icardo O, Bullido MJ, et al. Assessing the role of the TREM2 p.R47H variant as a risk factor for Alzheimer's disease and frontotemporal dementia. Neurobiol Aging 2014; 35: 444.e1-4.

10 Cuyvers E, Bettens K, Philtjens S, et al. Investigating the role of rare heterozygous TREM2 variants in Alzheimer's disease and frontotemporal dementia. Neurobiol Aging 2014; 35: 726.e11-9.

11 Reitz C, Mayeux R, Alzheimer's Disease Genetics Consortium. TREM2 and neurodegenerative disease. N Engl J Med 2013; 369: 1564-5.

$12 \mathrm{Yu}$ J-T, Jiang T, Wang Y-L, et al. Triggering receptor expressed on myeloid cells 2 variant is rare in late-onset Alzheimer's disease in Han Chinese individuals. Neurobiol Aging 2014; 35: 937.e1-3.

13 Ma J, Zhou Y, Xu J, et al. Association study of TREM2 polymorphism rs75932628 with lateonset Alzheimer's disease in Chinese Han population. Neurol Res 2014; 36: 894-6.

14 Jiao B, Liu X, Tang B, et al. Investigation of TREM2, PLD3, and UNC5C variants in patients with Alzheimer's disease from mainland China. Neurobiol Aging 2014; 35: 2422.e92422.e11.

15 Bonham LW, Sirkis DW, Fan J, et al. Identification of a rare coding variant in TREM2 in a Chinese individual with Alzheimer's disease. Neurocase 2017; 23: 65-9.

16 Miyashita A, Wen Y, Kitamura N, et al. Lack of genetic association between TREM2 and late-onset Alzheimer's disease in a Japanese population. J Alzheimers Dis 2014; 41: 10318. 
17 Chung SJ, Kim M-J, Kim J, et al. Exome array study did not identify novel variants in Alzheimer's disease. Neurobiol Aging 2014; 35: 1958.e13-4.

18 Mehrjoo Z, Najmabadi A, Abedini SS, et al. Association Study of the TREM2 Gene and Identification of a Novel Variant in Exon 2 in Iranian Patients with Late-Onset Alzheimer's Disease. Med Princ Pract 2015; 24: 351-4.

19 Hooli BV, Parrado AR, Mullin K, et al. The rare TREM2 R47H variant exerts only a modest effect on Alzheimer disease risk. Neurology 2014; 83: 1353-8.

20 Bertram L, Parrado AR, Tanzi RE. TREM2 and neurodegenerative disease. N Engl J Med 2013; 369: 1565.

21 Korvatska O, Leverenz JB, Jayadev S, et al. R47H Variant of TREM2 Associated With Alzheimer Disease in a Large Late-Onset Family: Clinical, Genetic, and Neuropathological Study. JAMA Neurol 2015; 72: 920-7.

22 Jiang $\mathrm{T}$, Tan $\mathrm{L}$, Chen $\mathrm{Q}$, et al. A rare coding variant in TREM2 increases risk for Alzheimer's disease in Han Chinese. Neurobiol Aging 2016; 42: 217.e1-3.

23 Schlepckow K, Kleinberger G, Fukumori A, et al. An Alzheimer-associated TREM2 variant occurs at the ADAM cleavage site and affects shedding and phagocytic function. EMBO Mol Med 2017; 9: 1356-65.

24 Thornton P, Sevalle J, Deery MJ, et al. TREM2 shedding by cleavage at the H157- S158 bond is accelerated for the Alzheimer's disease- associated $\mathrm{H} 157 \mathrm{Y}$ variant. EMBO Mol Med 2017; : e201707673.

25 Jin SC, Carrasquillo MM, Benitez BA, et al. TREM2 is associated with increased risk for Alzheimer's disease in African Americans. Mol Neurodegener 2015; 10: 19.

26 Lambert JC, Ibrahim-Verbaas CA, Harold D, et al. Meta-analysis of 74,046 individuals identifies 11 new susceptibility loci for Alzheimer's disease. Nat Genet 2013; 45: 1452-8.

27 Benitez BA, Jin SC, Guerreiro R, et al. Missense variant in TREML2 protects against Alzheimer's disease. Neurobiol Aging 2014; 35: 1510.e19-26.

28 Carrasquillo MM, Allen M, Burgess JD, et al. A candidate regulatory variant at the TREM gene cluster associates with decreased Alzheimer's disease risk and increased TREML1 and TREM2 brain gene expression. Alzheimers Dement 2017; 13: 663-73.

29 Slattery CF, Beck JA, Harper L, et al. R47H TREM2 variant increases risk of typical earlyonset Alzheimer's disease but not of prion or frontotemporal dementia. Alzheimers Dement 2014; 10: 602-8.e4.

30 Rosenthal SL, Bamne MN, Wang X, et al. More evidence for association of a rare TREM2 mutation (R47H) with Alzheimer's disease risk. Neurobiol Aging 2015; 36: 2443.e21-6.

31 Roussos P, Katsel P, Fam P, Tan W, Purohit DP, Haroutunian V. The triggering receptor expressed on myeloid cells 2 (TREM2) is associated with enhanced inflammation, neuropathological lesions and increased risk for Alzheimer's dementia. Alzheimers Dement 2015; 11: 1163-70. 
32 Luis EO, Ortega-Cubero S, Lamet I, et al. Frontobasal gray matter loss is associated with the TREM2 p.R47H variant. Neurobiol Aging 2014; 35: 2681-90.

33 Lill CM, Rengmark A, Pihlstrøm L, et al. The role of TREM2 R47H as a risk factor for Alzheimer's disease, frontotemporal lobar degeneration, amyotrophic lateral sclerosis, and Parkinson's disease. Alzheimers Dement 2015; 11: 1407-16.

34 Cruchaga C, Kauwe JSK, Harari O, et al. GWAS of cerebrospinal fluid tau levels identifies risk variants for Alzheimer's disease. Neuron 2013; 78: 256-68.

35 Piccio L, Deming Y, Del-Águila JL, et al. Cerebrospinal fluid soluble TREM2 is higher in Alzheimer disease and associated with mutation status. Acta Neuropathol 2016; 131: 92533.

36 Rajagopalan P, Hibar DP, Thompson PM. TREM2 and neurodegenerative disease. N Engl J Med 2013; 369: 1565-7.

37 Engelman CD, Koscik RL, Jonaitis EM, Hermann BP, La Rue A, Sager MA. Investigation of triggering receptor expressed on myeloid cells 2 variant in the Wisconsin Registry for Alzheimer's Prevention. Neurobiol Aging 2014; 35: 1252-4.

38 Replogle JM, Chan G, White CC, et al. A TREM1 variant alters the accumulation of Alzheimer-related amyloid pathology. Ann Neurol 2015; 77: 469-77.

39 Henjum K, Almdahl IS, Årskog V, et al. Cerebrospinal fluid soluble TREM2 in aging and Alzheimer's disease. Alzheimers Res Ther 2016; 8: 17.

40 Zhong L, Chen X-F, Wang T, et al. Soluble TREM2 induces inflammatory responses and enhances microglial survival. J Exp Med 2017; 214: 597-607.

41 Kleinberger G, Yamanishi $\mathrm{Y}$, Suárez-Calvet $\mathrm{M}$, et al. TREM2 mutations implicated in neurodegeneration impair cell surface transport and phagocytosis. Sci Transl Med 2014; 6: $243 r a 86$.

42 Heslegrave A, Heywood W, Paterson R, et al. Increased cerebrospinal fluid soluble TREM2 concentration in Alzheimer's disease. Mol Neurodegener 2016; 11: 3.

43 Suárez-Calvet M, Kleinberger G, Araque Caballero MÁ, et al. sTREM2 cerebrospinal fluid levels are a potential biomarker for microglia activity in early-stage Alzheimer's disease and associate with neuronal injury markers. EMBO Mol Med 2016; 8: 466-76.

44 Suárez-Calvet M, Araque Caballero MÁ, Kleinberger G, et al. Early changes in CSF sTREM2 in dominantly inherited Alzheimer's disease occur after amyloid deposition and neuronal injury. Sci Transl Med 2016; 8: 369ra178.

45 Ulrich JD, Ulland TK, Colonna M, Holtzman DM. Elucidating the Role of TREM2 in Alzheimer's Disease. Neuron 2017; 94: 237-48.

46 Brosseron F, Traschütz A, Widmann CN, et al. Characterization and clinical use of inflammatory cerebrospinal fluid protein markers in Alzheimer's disease. Alzheimers Res Ther 2018; 10: 25.

47 Colonna M, Wang Y. TREM2 variants: new keys to decipher Alzheimer disease 
pathogenesis. Nat Rev Neurosci 2016; 17: 201-7.

48 Yuan P, Condello C, Keene CD, et al. TREM2 Haplodeficiency in Mice and Humans Impairs the Microglia Barrier Function Leading to Decreased Amyloid Compaction and Severe Axonal Dystrophy. Neuron 2016; 90: 724-39.

49 Wang Y, Ulland TK, Ulrich JD, et al. TREM2-mediated early microglial response limits diffusion and toxicity of amyloid plaques. J Exp Med 2016; 213: 667-75.

50 Krasemann S, Madore C, Cialic R, et al. The TREM2-APOE Pathway Drives the Transcriptional Phenotype of Dysfunctional Microglia in Neurodegenerative Diseases. Immunity 2017; 47: 566-81.e9.

51 Song WM, Joshita S, Zhou Y, Ulland TK, Gilfillan S, Colonna M. Humanized TREM2 mice reveal microglia-intrinsic and -extrinsic effects of R47H polymorphism. J Exp Med 2018; 215: 745-60.

52 Jay TR, Hirsch AM, Broihier ML, et al. Disease Progression-Dependent Effects of TREM2 Deficiency in a Mouse Model of Alzheimer's Disease. J Neurosci 2017; 37: 637-47.

53 Wang $\mathrm{Y}$, Cella M, Mallinson K, et al. TREM2 lipid sensing sustains the microglial response in an Alzheimer's disease model. Cell 2015; 160: 1061-71.

54 Jay TR, Miller CM, Cheng PJ, et al. TREM2 deficiency eliminates TREM2+ inflammatory macrophages and ameliorates pathology in Alzheimer's disease mouse models. $J$ Exp Med 2015; 212: 287-95.

55 Lee CYD, Daggett A, Gu X, et al. Elevated TREM2 Gene Dosage Reprograms Microglia Responsivity and Ameliorates Pathological Phenotypes in Alzheimer's Disease Models. Neuron 2018; 97: 1032-48.e5.

$56 \mathrm{Ma} \mathrm{L}$, Allen M, Sakae N, et al. Expression and processing analyses of wild type and p.R47H TREM2 variant in Alzheimer's disease brains. Mol Neurodegener 2016; 11: 72.

57 Yin J, Liu X, He Q, Zhou L, Yuan Z, Zhao S. Vps35-dependent recycling of Trem2 regulates microglial function. Traffic 2016; 17: 1286-96.

58 Zhao Y, Wu X, Li X, et al. TREM2 Is a Receptor for $\beta$-Amyloid that Mediates Microglial Function. Neuron 2018; 97: 1023-31.e7.

59 Yeh FL, Wang Y, Tom I, Gonzalez LC, Sheng M. TREM2 Binds to Apolipoproteins, Including APOE and CLU/APOJ, and Thereby Facilitates Uptake of Amyloid-Beta by Microglia. Neuron 2016; 91: 328-40.

60 Bailey CC, DeVaux LB, Farzan M. The Triggering Receptor Expressed on Myeloid Cells 2 Binds Apolipoprotein E. J Biol Chem 2015; 290: 26033-42.

61 Atagi Y, Liu C-C, Painter MM, et al. Apolipoprotein E Is a Ligand for Triggering Receptor Expressed on Myeloid Cells 2 (TREM2). J Biol Chem 2015; 290: 26043-50.

62 Zajkowicz A, Gdowicz-Kłosok A, Krześniak M, Janus P, Łasut B, Rusin M. The Alzheimer’s disease-associated TREM2 gene is regulated by p53 tumor suppressor protein. Neurosci Lett 2018; published online May 26. DOI:10.1016/j.neulet.2018.05.037. 
63 Keren-Shaul H, Spinrad A, Weiner A, et al. A Unique Microglia Type Associated with Restricting Development of Alzheimer's Disease. Cell 2017; 169: 1276-90.e17.

64 Mazaheri F, Snaidero N, Kleinberger G, et al. TREM2 deficiency impairs chemotaxis and microglial responses to neuronal injury. EMBO Rep 2017; 18: 1186-98.

65 Ulland TK, Song WM, Huang SC-C, et al. TREM2 Maintains Microglial Metabolic Fitness in Alzheimer's Disease. Cell 2017; 170: 649-63.e13.

66 Leyns CEG, Ulrich JD, Finn MB, et al. TREM2 deficiency attenuates neuroinflammation and protects against neurodegeneration in a mouse model of tauopathy. Proc Natl Acad Sci U S A 2017; 114: 11524-9.

67 Jiang T, Tan L, Zhu X-C, et al. Silencing of TREM2 exacerbates tau pathology, neurodegenerative changes, and spatial learning deficits in P301S tau transgenic mice. Neurobiol Aging 2015; 36: 3176-86.

68 Bemiller SM, McCray TJ, Allan K, et al. TREM2 deficiency exacerbates tau pathology through dysregulated kinase signaling in a mouse model of tauopathy. Mol Neurodegener 2017; $12: 74$.

69 Pekkarinen P, Hovatta I, Hakola P, et al. Assignment of the locus for PLO-SL, a frontal-lobe dementia with bone cysts, to 19q13. Am J Hum Genet 1998; 62: 362-72.

70 Klünemann $\mathrm{HH}$, Ridha $\mathrm{BH}$, Magy $\mathrm{L}$, et al. The genetic causes of basal ganglia calcification, dementia, and bone cysts: DAP12 and TREM2. Neurology 2005; 64: 1502-7.

71 Chepuru R, Shaik AJ, Tandra SR, Gaddamanugu P, Alladi S, Kaul S. Nasu-Hakola disease (polycystic lipomembranous osteodysplasia with sclerosing leukoencephalopathy): First report from India. Neurol India 2018; 66: 538.

72 Paloneva J, Autti T, Hakola P, Haltia MJ. Polycystic Lipomembranous Osteodysplasia with Sclerosing Leukoencephalopathy (PLOSL). In: Adam MP, Ardinger HH, Pagon RA, et al., eds. GeneReviews®. Seattle (WA): University of Washington, Seattle, 2002.

73 Solje E, Hartikainen P, Valori M, et al. C9ORF72 expansion does not affect the phenotype in Nasu-Hakola disease with the DAP12 mutation. Neurobiol Aging 2014; 35: 1780.e13-7.

74 Ghezzi L, Carandini T, Arighi A, et al. Evidence of CNS $\beta$-amyloid deposition in NasuHakola disease due to the TREM2 Q33X mutation. Neurology 2017; : 10.1212/WNL.0000000000004747.

75 Montalbetti L, Ratti MT, Greco B, Aprile C, Moglia A, Soragna D. Neuropsychological tests and functional nuclear neuroimaging provide evidence of subclinical impairment in NasuHakola disease heterozygotes. Funct Neurol 2005; 20: 71-5.

76 Satoh J-I, Kino Y, Yanaizu M, Saito Y. Alzheimer's disease pathology in Nasu-Hakola disease brains. Intractable Rare Dis Res 2018; 7: 32-6.

77 Kaivola K, Jansson L, Saarentaus E, et al. Heterozygous TYROBP deletion (PLOSLFIN) is not a strong risk factor for cognitive impairment. Neurobiol Aging 2018; 64: 159.e1-159.e4.

78 Darwent L, Carmona S, Lohmann E, et al. Mutations in TYROBP are not a common cause 
of dementia in a Turkish cohort. Neurobiol Aging 2017; 58: 240.e1-240.e3.

79 Chouery E, Delague V, Bergougnoux A, Koussa S, Serre J-L, Mégarbané A. Mutations in TREM2 lead to pure early-onset dementia without bone cysts. Hum Mutat 2008; 29. http://onlinelibrary.wiley.com/doi/10.1002/humu.20836/full.

80 Guerreiro RJ, Lohmann E, Brás JM, et al. Using exome sequencing to reveal mutations in TREM2 presenting as a frontotemporal dementia-like syndrome without bone involvement. JAMA Neurol 2013; 70: 78-84.

81 Guerreiro R, Bilgic B, Guven G, et al. A novel compound heterozygous mutation in TREM2 found in a Turkish frontotemporal dementia-like family. Neurobiol Aging 2013; 34: 2890.e12890.e5.

$82 \mathrm{Ng}$ AS, Tan YJ, Yi Z, et al. Targeted exome sequencing reveals homozygous TREM2 R47C mutation presenting with behavioral variant frontotemporal dementia without bone involvement. Neurobiol Aging 2018; published online April 16.

DOI:10.1016/j.neurobiolaging.2018.04.003.

83 Le Ber I, De Septenville A, Guerreiro R, et al. Homozygous TREM2 mutation in a family with atypical frontotemporal dementia. Neurobiol Aging 2014; 35: 2419.e23-2419.e25.

84 Redaelli V, Salsano E, Colleoni L, et al. Frontotemporal Dementia and Chorea Associated with a Compound Heterozygous TREM2 Mutation. J Alzheimers Dis 2018; 63: 195-201.

85 Chee KY, Gaillard F, Velakoulis D, Ang CL, Chin LK, Ariffin R. A case of TREM2 mutation presenting with features of progressive non-fluent aphasia and without bone involvement. Aust N Z J Psychiatry 2017; 51: 1157-8.

86 Giraldo M, Lopera F, Siniard AL, et al. Variants in triggering receptor expressed on myeloid cells 2 are associated with both behavioral variant frontotemporal lobar degeneration and Alzheimer's disease. Neurobiol Aging 2013; 34: 2077.e11-8.

87 Thelen M, Razquin C, Hernández I, et al. Investigation of the role of rare TREM2 variants in frontotemporal dementia subtypes. Neurobiol Aging 2014; 35: 2657.e13-2657.e19.

88 Borroni B, Ferrari F, Galimberti D, et al. Heterozygous TREM2 mutations in frontotemporal dementia. Neurobiol Aging 2014; 35: 934.e7-10.

89 Rayaprolu S, Mullen B, Baker M, et al. TREM2 in neurodegeneration: evidence for association of the p.R47H variant with frontotemporal dementia and Parkinson's disease. Mol Neurodegener 2013; 8: 19.

90 Lattante S, Le Ber I, Camuzat A, et al. TREM2 mutations are rare in a French cohort of patients with frontotemporal dementia. Neurobiol Aging 2013; 34: 2443.e1-2.

91 Benitez BA, Cruchaga C, United States-Spain Parkinson's Disease Research Group. TREM2 and neurodegenerative disease. N Engl J Med 2013; 369: 1567-8.

92 Guerreiro R, Hardy J. TREM2 and neurodegenerative disease. N Engl J Med 2013; 369: 1569-70.

93 Mengel D, Thelen M, Balzer-Geldsetzer M, et al. TREM2 rare variant p.R47H is not 
associated with Parkinson's disease. Parkinsonism Relat Disord 2016; 23: 109-11.

94 Feng S-J, Nie K, Gan R, et al. Triggering receptor expressed on myeloid cells 2 variants are rare in Parkinson's disease in a Han Chinese cohort. Neurobiol Aging 2014; 35: 1780.e11-2.

95 Chen Y, Chen X, Guo X, et al. Assessment of TREM2 rs75932628 association with Parkinson's disease and multiple system atrophy in a Chinese population. Neurol Sci 2015; 36: 1903-6.

96 Tan T, Song Z, Yuan L, et al. Genetic analysis of TREM2 variants in Chinese Han patients with sporadic Parkinson's disease. Neurosci Lett 2016; 612: 189-92.

97 Li Z, Zhong L, Gu L, et al. Association study of TREM2 polymorphism rs75932628 with leucoaraiosis or Parkinson's disease in the Han Chinese population. BMJ Open 2016; 6: e009499.

98 Cady J, Koval ED, Benitez BA, et al. TREM2 variant p. R47H as a risk factor for sporadic amyotrophic lateral sclerosis. JAMA Neurol 2014; 71: 449-53.

99 Chen X, Chen Y, Wei Q, et al. Assessment of TREM2 rs75932628 association with amyotrophic lateral sclerosis in a Chinese population. J Neurol Sci 2015; 355: 193-5.

100 Walton RL, Soto-Ortolaza AI, Murray ME, et al. TREM2 p.R47H substitution is not associated with dementia with Lewy bodies. Neurol Genet 2016; 2: e85.

101 Carrasquillo MM, Barber I, Lincoln SJ, et al. Evaluating pathogenic dementia variants in posterior cortical atrophy. Neurobiol Aging 2016; 37: 38-44.

102 Ortega-Cubero S, Lorenzo-Betancor O, Lorenzo E, et al. TREM2 R47H variant and risk of essential tremor: a cross-sectional international multicenter study. Parkinsonism Relat Disord 2015; 21: 306-9.

103 Jay TR, von Saucken VE, Landreth GE. TREM2 in Neurodegenerative Diseases. Mol Neurodegener 2017; 12: 56.

104 Ito $\mathrm{H}$, Hamerman JA. TREM-2, triggering receptor expressed on myeloid cell-2, negatively regulates TLR responses in dendritic cells. Eur J Immunol 2012; 42: 176-85.

105 Wunderlich P, Glebov K, Kemmerling N, Tien NT, Neumann H, Walter J. Sequential proteolytic processing of the triggering receptor expressed on myeloid cells-2 (TREM2) protein by ectodomain shedding and $\mathrm{y}$-secretase-dependent intramembranous cleavage. $J$ Biol Chem 2013; 288: 33027-36.

106 Feuerbach D, Schindler P, Barske C, et al. ADAM17 is the main sheddase for the generation of human triggering receptor expressed in myeloid cells ( $h T R E M 2)$ ectodomain and cleaves TREM2 after Histidine 157. Neurosci Lett 2017; 660: 109-14.

\section{Panel: TREM2 and the TREM locus}




\section{TREM locus}

TREM2 is located within a gene cluster on chromosome 6p21.1, near TREM1, TREML1, TREML2, TREML3P, TREML4, and NCR2. The genes in this cluster have significant homology and are mostly involved in immunological functions.

\section{TREM2 expression}

The triggering receptor expressed on myeloid cells 2 (TREM2) is a transmembrane receptor of the immunoglobulin superfamily expressed in cells of the myeloid lineage, including microglia and osteoclasts, that participates in modulation of the immune system ${ }^{47,103}$. In the CNS the signal intensity levels of TREM2 transcripts are strongest in the basal ganglia, corpus callosum, medulla oblongata, and spinal cord ${ }^{4}$.

\section{TREM2 ligands and signaling cascade}

TREM2 binds anionic ligands including bacterial lipopolysaccharides, DNA and phospholipids ${ }^{53}$. Ligand binding to TREM2 initiates a signaling cascade in which the TREM2-associated intracellular adaptor TYROPB (or DAP12) is phosphorylated. Phosphorylated TYROBP recruits the protein tyrosine kinase SYK, which in turn induces PI3K activation, followed by AKT activation, MAPK activation, $\mathrm{Ca}^{2+}$ mobilization, and other downstream effects ${ }^{47}$. Activation of these pathways modulates cell proliferation and differentiation, survival, phagocytosis, chemotaxis, and inflammation ${ }^{47103}$. In certain contexts, TREM2 signaling can also be inhibitory, with a negative regulation of TLR-response found to be mediated by TREM2 in dendritic cells 104 .

\section{TREM2 cleavage and soluble TREM2}

TREM2, a type I membrane glycoprotein, can be subjected to sequential proteolytic processing. Cleavage by alpha secretases (ADAM10 and ADAM17) occurring after the $\mathrm{H} 157$ residue of the protein, results in ectodomain shedding, releasing soluble TREM2 into the extracellular space 105. Gamma-secretase then cleaves teh remaining C-terminal portion ${ }^{23,24,106}$. 